Background Trichomonas vaginalis (TV) infection is a prevalent parasitic STD that may increase the risk of acquiring other STI including HIV. Trichomoniasis has been increasingly reported in older female populations. Detection methods include the InPouch culture system and GenProbe's APTIMA Trichomonas vaginalis nucleic acid amplification test (NAAT). Both systems detect TV (viable or genetic material, respectively) from vaginal swabs. In addition to comparing TV detection technologies, this study attempted to look at infection status in younger versus older macaque populations.

Methods 24 sexually mature female pigtailed macaques were challenged with TV (ATCC 50148, human isolate), and followed weekly for five weeks, before metronidazole treatment. The reproductive age span for pigtailed macaques is approximately 4 to 18 years of age. Twelve animals aged 4-7 and twelve animals older than 13 years were enrolled. Paired vaginal swabs were collected weekly from each animal for culture and NAAT detection assays.

Results Each TV-challenged macaque developed trichomoniasis. Of 199 matched samples (culture and NAAT), 13 had discrepant results. Six of these were likely due to false culture results. Four samples represented the transition time from positive to negative status in three animals. It is plausible that organisms detected by NAAT were no longer viable (thus culture-negative). There is no obvious explanation for the three remaining discrepant results.

Trichomoniasis infection was self-limited (resolved prior to metronidazole treatment) in eight animals: two older and six younger macaques. Two of these younger macaques experienced intermittent discrepant results after testing negative by both methods for two consecutive weeks.

Conclusions NAAT detection appears to be more sensitive and less prone to erroneous results in this laboratory's experience. There may be a trend for younger animals to self-clear TV infection faster than older animals, which might explain the increased TV infection rates noted in older women.

\section{P1.044 A CROSS-SECTIONAL STUDY COMPARING SERUM VITAMIN D LEVELS IN HUMAN IMMUNODEFICIENCY VIRUS-INFECTED AND -UNINFECTED INDIVIDUALS}

doi:10.1136/sextrans-2013-051184.0264

F Yousefi, F Moradpour. Ahwaz Jundishapur University of Medical Sciences, Ahwaz, Iran

Background Since the evolution of human immunodeficiency virus (HIV) infection, every effort has been made to increase the survival and quality of life of the infected patients. In this regard, metabolic/nutritional derangements are among the most important problems. So, we planned this study to evaluate the serum level of vitamin $\mathrm{D}$ as an essential micronutrient with known immunologic roles in HIV-infected patients and compare it with that of HIVuninfected individuals, and to identify risk factors for possible hypovitaminosis $\mathrm{D}$ in the former group.

Methods This was a cross sectional study on 35 HIV-infected patients (cases) and 35 HIV-uninfected individuals (controls). All the participants were $>=18$ years-old. Patients with conditions, like tuberculosis, or using drugs, with known effects on serum vitamin $\mathrm{D}$ level were excluded. The control group was matched for age, sex, nutritional habits and occupation (exposure to sunlight). HIV infection was confirmed in the cases with 2 positive HIV ELISAs and then a positive HIV Western Blot test. Serum level of vitamin D was measured by ELISA method. Chi-square and independent $\mathrm{T}$ tests were used for analysis of data.

Results Of the HIV-infected patients, 9 people (25.7\%) were receiving antiretroviral therapy. Twenty-three HIV-infected patient $(65.7 \%)$ had hypovitaminosis D (vitamin $\mathrm{D}<30 \mathrm{ng} / \mathrm{mL}$ ), comparing with only one person $(2.8 \%)$ in controls ( $p$ value $<0.05)$. The mean level of vitamin $\mathrm{D}$ in the serum was significantly lower in HIVinfected patients $(25.78 \mathrm{ng} / \mathrm{mL})$ compared with HIV- uninfected individuals $(41.4 \mathrm{ng} / \mathrm{mL})(\mathrm{p}$ value $<0.008)$. There was no association between hypovitaminosis $\mathrm{D}$ and sex, age, body mass index, CD4+ cell count, haemoglobin level and antiretroviral therapy in HIVinfected patients.

Conclusion This study shows the possible association of HIVinfection with vitamin D deficiency, and thus the evaluation of this group of patients for hypovitaminosis $\mathrm{D}$ seems reasonable.

\section{P1.045 ZINC IONOPHORES INHIBIT HERPES SIMPLEX VIRUS TYPE 1 AND 2 REPLICATION THROUGH DYSREGULATION OF UBIQUITIN-PROTEASOME PATHWAY}

doi:10.1136/sextrans-2013-051184.0265

'M Oiu, 'Y Chu, 'H Song, ${ }^{1,2 Z}$ Wu. 'Center for Public Health Research, Nanjing University, Nanjing, China; ${ }^{2}$ State Key Lab of Analytical Chemistry for Life Science, Nanjing University, Nanjing, China

Background Herpes simplex virus types 1 and 2 (HSV-1 and HSV-2) are among the most prevalent human pathogens in both industrialised and developing regions. In the current study, we reported that two zinc ionephores, pyrrolidine dithiocarbamate (PDTC) and pyrithione (PT) could inhibit herpes simplex virus type 1 and type 2 (HSV-1 and HSV-2) replication.

Methods Viral replication was evaluated via detecting HSV late gene product (Glycoprotein, gD) using In-cell Western. NF- $\mathrm{BB}$, MAPK activation, viral immediate-early genes expression and profile of cellular ubiquitin-conjugates were determined by Western blot. Protein localization was investigated via immunofluorescence staining. Viral protein expression on mRNA level was quantified by realtime-PCR. 26S Proteasome activation was determined using fluorogenic substrate, Suc-LLVY-AMC.

Results PDTC and PT inhibited HSV-1 and HSV-2 gD expression and the production of viral progeny, which was dependent on zinc ion. Further studies showed that these two compounds suppressed the HSV immediate-early gene, the infected cell polypeptide 4 (ICP4) expression, but had less effect on ICP0. HSV infection could interact with cellular ubiquitin-proteasome system (UPS) and cause loss of high molecular weight ubiquitin-conjugates. It was found that PDTC and PT could interfere UPS, leading to the inhibition of HSV-2-induced I $\mathrm{KB}-\alpha$ degradation to prevent NF- $\kappa \mathrm{B}$ activation and enhanced PML stability in nucleus. However, PDTC and PT did not show direct inhibition of $26 \mathrm{~S}$ proteasome activity. Instead, these two zinc ionphores induced import of extracellular zinc ions into cells, which facilitated dysregulation of proteasome function and accumulation of intracellular ubiquitinconjugates. Other evidence was that the inhibitors of ubiquitin activating enzyme E1 and deubiquitinase also inhibited HSV replication, implying that UPS was required for effective replication of HSV-1 and HSV-2

Conclusion Homostasis of ubiquitin cycle and UPS were critical for HSV gene expression and replication and that the ubiquitinproteasome pathway is a potential drug target for HSV infection.

\section{P.02 - Clinical Sciences Track}

\section{P2.001 PERFORMANCE OF THE HOLOGIC GEN-PROBE APTIMA

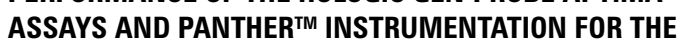 CONFIRMATION OF NEISSERIA GONORRHOEAE IN GENITAL AND NON-GENITAL SAMPLES}

doi:10.1136/sextrans-2013-051184.0266

'P Lowe, ${ }^{2} \mathrm{~S}$ Dubedat, ${ }^{3} \mathrm{M}$ Turra. ${ }^{1}$ Hologic Australia, Macquarie Park, Australia; ${ }^{2}$ Royal Prince Alfred Hospital, Sydney, Australia; ${ }^{3}$ SA Pathology - IMVS, Adelaide, Australia 
Nucleic Acid Amplification Tests (NAATs) for Chlamydia trachomatis (CT) and Neisseria gonorhoeae (GC) have become routine. These methods are validated for use with urogenital samples and have a faster turn-around-time for results with automation. Testing of non-validated samples is common which raises concerns about assay performance. In Australia, the 2005 Public Health Laboratory Network (PHLN) guideline recommends repeat testing of all initial positive GC NAAT results with a suitable alternate NAAT assay. In this study, results from $>70,000$ patient samples from 2 laboratories were reviewed in accordance with the PHLN recommendations. The laboratories used the APTIMA Combo 2 (AC2) and APTIMA Neisseria gonorrhoeae (AGC) assays with the PANTHER instrument.

72,253 AC2 results were available for analysis which included $1,174(1.68 \%)$ initially AC2 reactive (positive or equivocal) samples which also had results for the AGC assay used as the confirmatory assay. For the reactive samples, the agreement between the AC2 and AGC assays occurred in $97.19 \%$ of samples; 1135 were positive in both assays and 6 were equivocal in both assays. Sample types tested included those with manufacturer's validation claims; urine, ThinPrep, vaginal, endocervical and urethral swabs and non-validated samples including throat, rectal, eye and joint fluids. Samples from throat swabs showed the highest numbers of discordant results followed by rectal swab samples. The percentage agreement of results obtained from all sample types was excellent, with an overall PPV of $>99 \%$.

Without confirmatory testing, false positive results would have been reported for 13 samples representing $0.02 \%$ of all samples tested. This study demonstrates that the initial AC2 results can be accepted with high confidence.

\section{P2.002 EVALUATION OF A REAL-TIME PCR-BASED TEST FOR BACTERIAL VAGINOSIS}

doi:10.1136/sextrans-2013-051184.0267

${ }^{1}$ T Ivanova, ${ }^{2} \mathrm{~K}$ Shalepo, ${ }^{2} \mathrm{~V}$ Nazarova, ${ }^{2} \mathrm{E}$ Shipitsyna, ${ }^{1} \mathrm{~A}$ Guschin, ${ }^{2} \mathrm{~A}$ Savicheva. ${ }^{1} \mathrm{Central}$ Research Institute for Epidemiology, Moscow, Russian Federation; ${ }^{2} D .0$. Ott Research Institute of Obstetrics and Gynaecology, St.Petersburg, Russian Federation

Background Bacterial vaginosis (BV) is the most common cause of vaginal discharge in women. Since BV is associated with significant morbidity, accurate tools for diagnosing the disease are important. The Amsel criteria (AC) and Nugent score (NS) are currently used for BV diagnosis. Recently, a number of PCR-based tests providing objective, sensitive and specific BV detection have been described. This study aimed to evaluate a newly developed BV test based on quantitative detection of Gardnerella vaginalis, Atopobium vaginae, Lactobacillus spp. and total Bacteria using multiplex PCR.

Methods PCR criteria were elaborated based on the relative counts of the targeted bacteria to classify vaginal microflora as BV, no BV or intermediate. Vaginal samples for the test evaluation were obtained from 274 patients addressing a gynaecologist for routine examination. All participants were of reproductive age, not pregnant and not menstruating at the time of enrollment. BV was diagnosed using the AC and NS.

Results According to the NS results, 66 patients were BV positive, 156 were BV negative, and 52 were classified as intermediate. All patients positive by the NS were positive by the AC, and all patients negative by the NS were negative by the AC. Among $66 \mathrm{BV}$ positive samples, 62 demonstrated PCR results corresponding to BV, and 4 samples were BV negative by PCR (94\% sensitivity). Of the 156 negative samples, 151 were interpreted as BV negative using PCR criteria, and 5 samples - as BV positive ( $97 \%$ specificity).

Conclusion A multiplex real-time PCR test for BV was developed showing $94 \%$ sensitivity and $97 \%$ specificity.

\section{P2.003 FEASIBILITY AND ACCEPTABILITY OF SELF-COLLECTED VAGINAL SWABS FOR DIAGNOSIS OF BACTERIAL VAGINOSIS AMONG PREGNANT WOMEN IN A COMMUNITY SETTING IN RURAL MYSORE, INDIA}

doi:10.1136/sextrans-2013-051184.0268

${ }^{1,2} \mathbf{P}$ Madhivanan, ${ }^{2} \mathrm{~K}$ Ravi, ${ }^{1} \mathrm{M}$ Wilcox, ${ }^{2} \mathrm{~B}$ Niranjankumar, ${ }^{2} \mathrm{R}$ Shaheen, ${ }^{2} \mathrm{~V}$ Srinivas, ${ }^{2} \mathrm{~A}$ Arun, ${ }^{2 P}$ Jaykrishna, ${ }^{2,1} \mathrm{~K}$ Krupp. ${ }^{1}$ Robert Stempel College of Public Health \& Social Work, Florida International University, Miami, FL, United States, ${ }^{2}$ Public Health Research Institute of India, Mysore, India

Background Bacterial vaginosis (BV) is a common cause of adverse birth outcomes. Its association with other obstetric and gynecologic complications and HIV are increasingly recognised. This study examined the acceptability and feasibility of using self-collected vaginal swabs (SVS) for screening of BV in a community setting among rural pregnant women in Mysore Taluk, India.

Methods A community based cross-sectional study was carried out between June 2007 and December 2010. Mobile medical clinics offered antenatal care and HIV testing in all 144 rural villages in Mysore Taluk. Women were also screened for BV using SVS and asked about their experience with the collecting process. Gramstain evaluation of vaginal samples using Nugent score (NS) was conducted by two trained laboratory personnel.

Results Among the 1675 women attending the mobile medical clinics from the 144 villages, 1541(92\%) were included in the analyses. 1639 agreed to provide vaginal swabs ( $97.8 \%$ response rate). The quality of the swabs was satisfactory in 1545 of the cases. There were 134 non-interpretable slides owing to poor quality. The median age of women was 20 years (range 14 to 40 years). Majority (98.7\%) reported themselves as Hindus and 1634 (97.5\%) were housewives. The prevalence of BV was $9.9 \%$ with a NS of 7-10 and $14.9 \%$ of women had intermediate flora on Nugent score of 4-6. While 212 women (12.9\%) reported collecting the vaginal swab as being 'very easy', $1402(85 \%)$ found it 'easy' and $22(1.3 \%)$ reported it as 'difficult'. Only 12 women were unable to collect the swab for various reasons.

Conclusion These study results support the use of self-collected vaginal swabs for diagnosing BV. Self-collected swabs to detect BV were well accepted by most of rural pregnant women in this region, and the quality of the swabs seemed to be satisfactory.

\section{P2.004 DOES BACTERIAL VAGINOSIS HAVE AN ASSOCIATION WITH SIL?}

doi:10.1136/sextrans-2013-051184.0269

D Ajit, S Gavas, S Joseph, S Kane, K Deodhar, B Rekhi, S Menon, A Budukh. Tata Memorial Hospital, Mumbai, India

Background Literature documents incomplete and conflicting evidence between bacterial vaginosis and its association with cervical intraepithelial neoplasia in cervicovaginal smears (CS).

Aim To investigate if there is an association between bacterial vaginosis (BV) and squamous intraepithelial lesion (SIL) in CS.

Methods A retrospective analysis of 6902 cervicovaginal cases accrued during January 2006 -December 2009 from an opportunistic screening programme was done. The cases diagnosed as unsatisfactory $(n=239)$, glandular cell abnormalities $(n=9)$ and carcinoma $(n=12)$ were excluded. Thus the study was based on 6642 cases. These were reviewed to identify SIL and BV. The correlation with histology was done wherever available. A diagnosis of BV was rendered if 10 clue cells per low power field and at least 5 fields showing cocco-bacteria were detected on Pap stained smears. Statistical evaluation was carried by applying $\mathrm{Z}$ test to determine if $\mathrm{BV}$ has an association to SIL in CS.

Results Of the 6642 cases analysed, BV was present in $13 \%$ cases and absent in $87 \%$ cases. SIL was reported in $7 \%$ BV present group and $2 \%$ in the $\mathrm{BV}$ absent group $(\mathrm{P}<0.001)$. The difference between 